\title{
Contrast-enhanced ultrasound in the therapeutic assessment of diffuse large B-cell lymphoma: A case report
}

\author{
WEI DU ${ }^{1 *}$, WENWU LING ${ }^{2 *}$, XUELEI MA ${ }^{3 *}$, CHONG JIANG $^{4}$, \\ JIANCHAO WANG ${ }^{1}$, CHENJIN ZHU $^{1}$ and XUEMING XIA ${ }^{5}$ \\ ${ }^{1}$ West China School of Medicine; Departments of ${ }^{2}$ Ultrasound and ${ }^{3}$ Medical Oncology, Cancer Center, \\ State Key Laboratory of Biotherapy; Departments of ${ }^{4}$ Nuclear Medicine and ${ }^{5}$ Medical Oncology, \\ West China Hospital, Sichuan University, Chengdu, Sichuan 610041, P.R. China
}

Received July 12, 2016; Accepted July 17, 2017

DOI: $10.3892 / \mathrm{ol} .2017 .6758$

\begin{abstract}
Contrast-enhanced computed tomography (CECT) has been extensively used in the restaging and assessment of treatment response for diffuse large B-cell lymphoma (DLBCL). However, CECT does not provide information regarding the specific functionality of lesions. A patient (56 years old, female) was previously admitted to the present institution, with bilateral cervical masses. Following numerous cycles of chemotherapy, a stable disease status was confirmed using CECT. In conjunction with CECT imaging results, contrast-enhanced ultrasound (CEUS) demonstrated important semi-functional information regarding blood perfusion, during the revision of treatment assessment. ${ }^{18}$ F-fluoro-2-deoxyglucose (FDG)-positron emission tomography-computed tomography imaging demonstrated no increase in FDG uptake of the same tumor lesion, consistent with the results of CEUS. CEUS exhibited the potential to present complementary results to CECT, in the therapeutic assessment of DLBCL, which, to the best of our knowledge, has not previously been reported.
\end{abstract}

\section{Introduction}

Diffuse large B-cell lymphoma (DLBCL) is the most frequently observed subtype of non-Hodgkin's lymphoma (1). Although DLBCL is aggressive, $60-80 \%$ of patients with DLBCL can be cured by using combination chemotherapy consisting of cyclophosphamide, doxorubicin, vincristine, and prednisone (CHOP), and anti-CD20 monoclonal antibody rituximab (R) (2). Nevertheless, $30-40 \%$ of patients show

Correspondence to: Dr Xuelei Ma, Department of Medical Oncology, Cancer Center, State Key Laboratory of Biotherapy, West China Hospital, Sichuan University, 37 GuoXue Alley, Chengdu, Sichuan 610041, P.R. China

E-mail: drmaxuelei@gmail.com

*Contributed equally

Key words: contrast-enhanced ultrasound, diffuse large B-cell lymphoma, imaging disease progression or relapse after a good initial response. It is important to accurately identify the patients who are at high risk of relapse as early as possible, so that they can be treated with alternative approaches.

${ }^{18}$ F-fluoro-2-deoxyglucose-positron emission tomography $\left({ }^{18} \mathrm{~F}-\mathrm{FDG}-\mathrm{PET}\right)$-computed tomography $\left({ }^{18} \mathrm{~F}-\mathrm{FDG}-\mathrm{PET} / \mathrm{CT}\right)$ provides information of the metabolic activity of lesions, has been utilized in the initial staging of malignant lymphomas, and is an accepted tool of response assessment after the end of the treatment (3-7). However, high rates of false positivity and other disadvantages for younger patients undergoing serial examinations mostly in metropolitan tertiary cancer centers, including radiation exposure and high costs, limits its application (8). Therefore, in the practically clinical course, CECT has been widely used in the restaging and assessment of the treatment response.

CEUS provides complementary information to CECT scan in the detection of blood perfusion of tumors (9-11). For the first time, we report a case of DLBCL, for which CEUS yielded important information on the response to treatment as well as guided the treatment. Informed consent was obtained from the patient for publication of this report and the accompanying images. As this is a case report, institutional review board approval was not necessary.

\section{Case report}

A 56-year-old woman was admitted to our department with bilateral cervical masses. Physical examination revealed several bilaterally enlarged lymph nodes in the neck. Nasopharyngolarygnoscopy showed a blood scab covering the Kiesselbach's plexus and angiectasis covering the right side. In addition, a neoplasm partly covered with a pseudomembrane was observed at the right side of the tonsil. Subsequently, a biopsy of the right-sided cervical mass showed CD2(+), CD3(+), CD20(+), Ki67+(30\%), Bcl-2(+), PAX-5(+), MUM1(+), CD79a(+), CD21(+) and FISH:EBER (-) (Fig. 1A-C). After discussing options, the patient chose deferred treatment and left the hospital. Two months later, the patient presented again with the aggressively growing mass in the right neck, accompanied by apparent pain. Therefore, the patient was referred to the nuclear medicine department 
for staging ${ }^{18} \mathrm{~F}-\mathrm{FDG}$ PET/CT (Fig. 1D-F). PET/CT showed an abnormally increased uptake of ${ }^{18} \mathrm{~F}-\mathrm{FDG}$ in the right side of the palatine tonsil; the cervical, thoracic, and iliac lymph nodes; and the bone and muscle of the left hipbone. Mild uptake of FDG was detected in the left side of the palatine tonsil. The maximum standardized uptake value (SUVmax) and the size of various nodal lesions are detailed in Table I. The patient was diagnosed with DLBCL (Ann Arbor stage IVA) based on the pathologic, and imaging findings.

Two cycles of standard R-CHOP chemotherapy (between April 23 and May 19, 2014) were immediately administered (Fig. 2). Considering the patient's economic burden, in order to assess the treatment response, we used CECT, broadly used in clinical practice, instead of PET/CT. A good clinical response was confirmed with CECT, which showed a reduced diameter of the largest cervical lymph node to be approximately $2.2 \mathrm{~cm}$ (Table I). The neck pain disappeared quickly. A partial response (PR) was confirmed, and the chemotherapy regimen was continued. After cycle four (between June 20 and July 18, 2014) (Fig. 2), stable disease (SD) status was confirmed using CECT, which showed that the largest diameter of the enlarged cervical lymph node did not decrease (Table I, Fig. 3), indicating that the tumors were not sensitive enough to R-CHOP. Therefore, we added etoposide to the treatment regimen, which became more intensive but also more toxic. Early studies concerning the practical use of CEUS in hepatic lymphoma and spermatic cord $(12,13)$ showed it could be used to get functional information on the blood perfusion of tumor lesions, compensating for the shortcomings of CECT. Therefore, after one cycle of chemotherapy with etoposide added to R-CHOP (R-CHOPE), we tried CEUS to evaluate the blood perfusion at the cervical lesions, which presumably partly represented the therapeutic effect in the whole body. Inconsistent with the CECT results, CEUS detected no blood flow at the biggest lymph node. After $1.2 \mathrm{ml}$ sulfur hexafluoride microbubble contrast medium (SonoVue, Bracco) was injected intravenously followed by a $15 \mathrm{ml}$ saline flush, CEUS showed no enhancement in the focal lesion for both the arterial and venous phases (Fig. 4A). This confirmed the lack of blood flow in the enlarged lymph node. This inconsistent result might indicate a better treatment response than we initially expected and necessitated revision of the previous treatment assessment. In order to address the reliability of this inconsistency, a PET/CT was performed, which showed that the increased uptake of lymph nodes in the palatine tonsil, neck, ilia, and hip had disappeared; the only high uptake presented in the thoracic lymphoma with a SUVmax of 4.8 in the whole body (Table I, Fig. 4B-E). We concluded that the uptake in the thoracic lymph node was due to an inflammatory lesion and the treatment response was revised to complete response (CR). The R-CHOPE regimen was switched to the less toxic R-CHOP regimen. After completing the sixth cycle of R-CHOP, the patient was discharged from the hospital. Three months later, no masses could be detected by palpation and no enhancement was detected by CEUS in all phases. The PET/CT performed in December confirmed this result, which showed no increased uptake in the right cervical lymph node, and no residual tumor was found in the whole body (Fig. 4B-E). The CECT performed in April 2015 showed no relapse in the whole body.

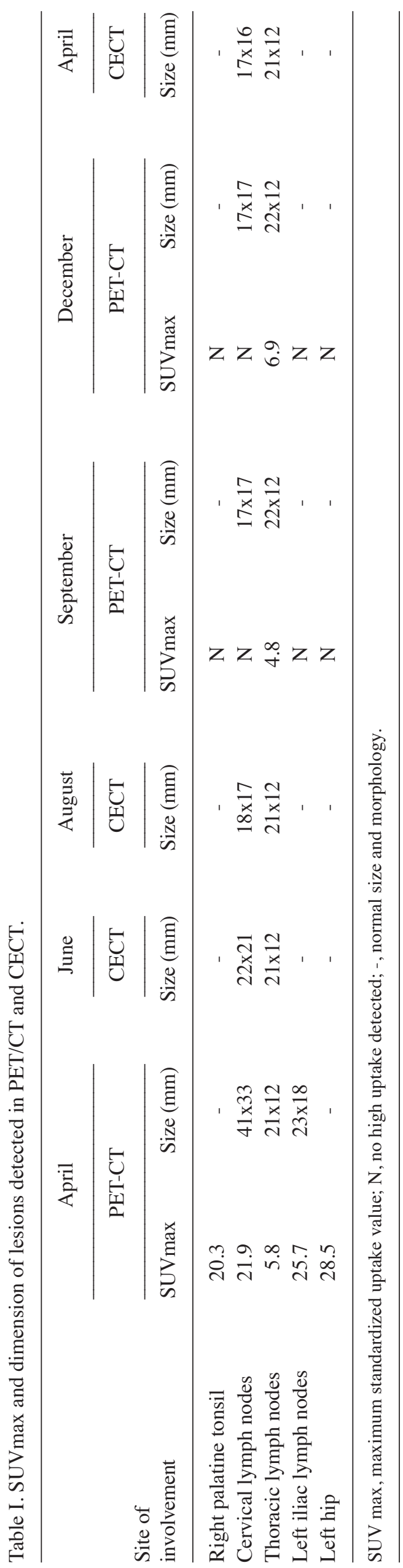


A

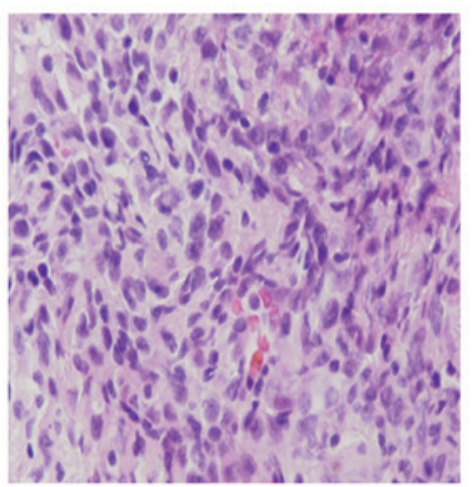

$\mathrm{D}$

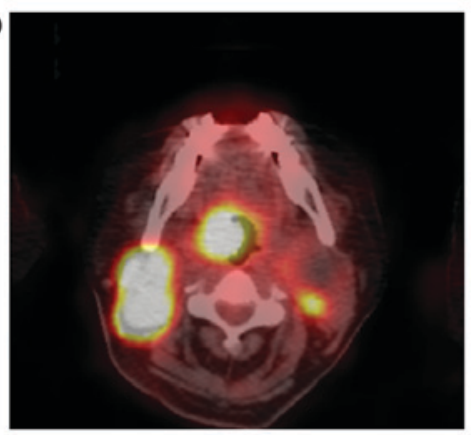

$\mathrm{B}$
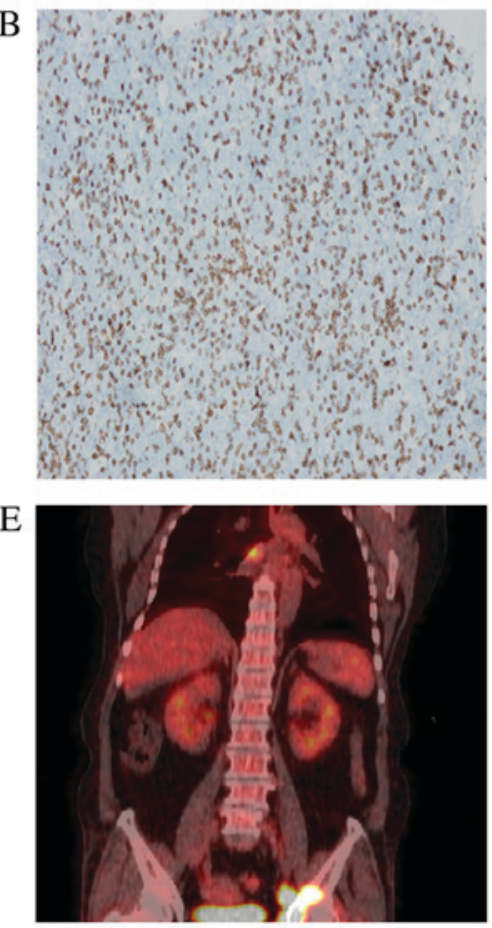

C
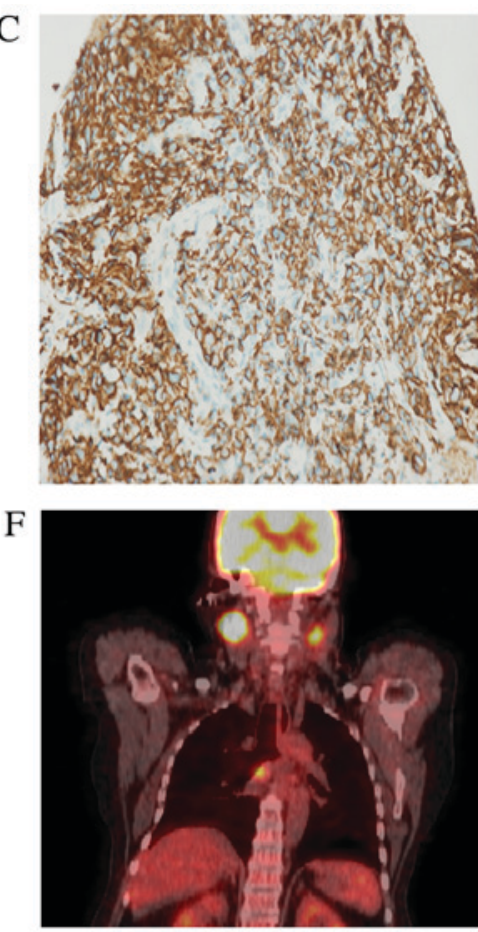

Figure 1. (A-C) Histopathological findings of the cervical lymph node biopsy specimen. (A) Large cells on H \& E stain (x200); (B) CD20 (+) cells (x100) and (C) (CD3 (+) cells (x100). (D-F) Coronal positron emission tomography-computed tomography (PET/CT) image showed increased ${ }^{18}$ F-FDG uptake at the right side of palatine tonsil; the cervical, thoracic, and iliac lymph nodes; and the bone and muscle of the left hipbone. Mild uptake of FDG was detected in the left side of palatine tonsil (A). Transaxial PET/CT image showed focally increased ${ }^{18} \mathrm{~F}$-FDG uptake in the cervical lesion (B). Findings were suggestive of lymphomatous involvement of the above mentioned sites.

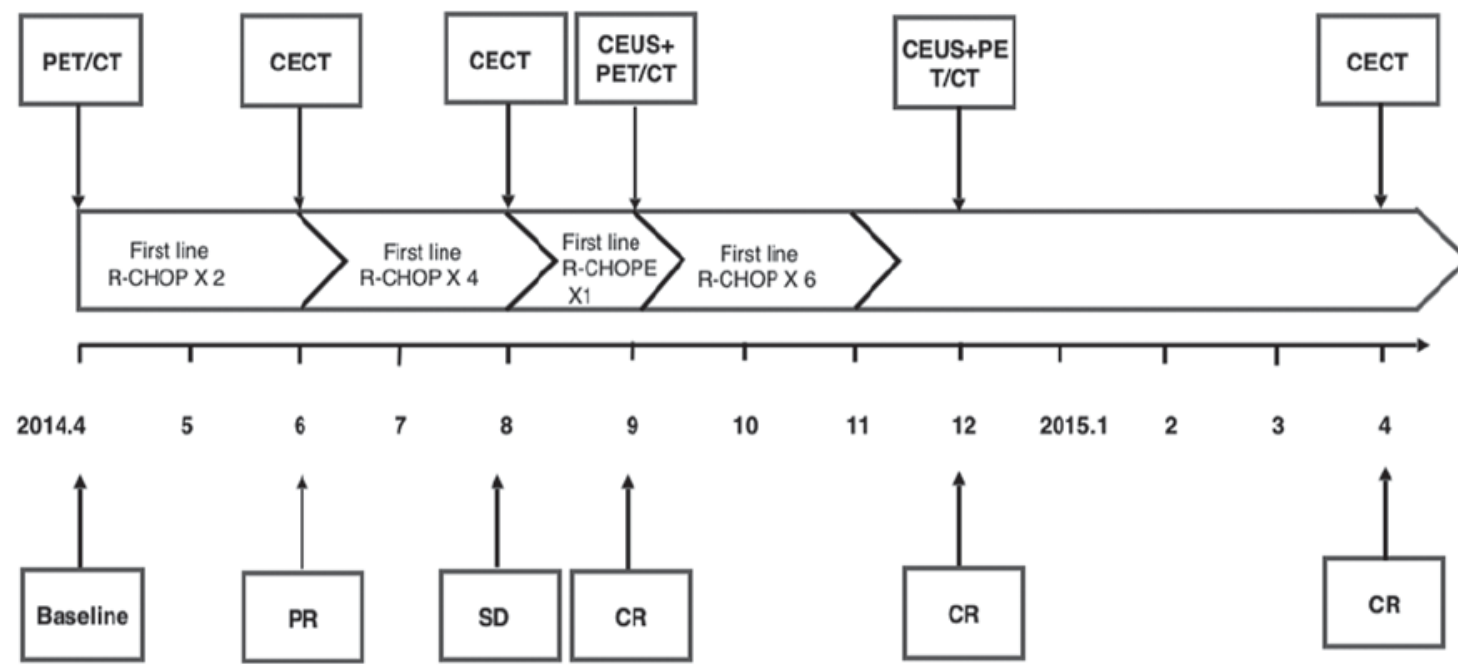

Figure 2. Treatment Schema. CR, complete response; PET/CT, positron emission tomography-computed tomography; CECT, contrast-enhanced computed tomography; CEUS, contrast-enhanced ultrasound; PR, partial response; SD, stable disease; CR, complete response; R-CHOP, rituximab plus cyclophosphamide, doxorubicin, vincristine, and prednisone; R-CHOPE, rituximab plus cyclophosphamide, doxorubicin, vincristine, prednisone etoposide, methylprednisolone, cisplatin, and cytarabine.

\section{Discussion}

Although DLBCL is one of the curable lymphomas, 20-40\% of patients experience relapse (2). Early treatment response assessment, a strategy for predicting which patients are likely to respond well to treatment, has gained importance in recent years (14-16). When a patient does not respond well enough to current chemotherapy, more aggressive treatment regimens including intense chemotherapy and autologous stem cell transplantation should be considered $(17,18)$.

Switching the treatment model from first-line to second-line therapy means undergoing more cycles of chemotherapy and imaging radiation exposure, longer hospitalization period, and a heavier economic burden.

Although the benefits of PET/CT clearly outweigh its detrimental effects, interim PET/CT for response assessment 

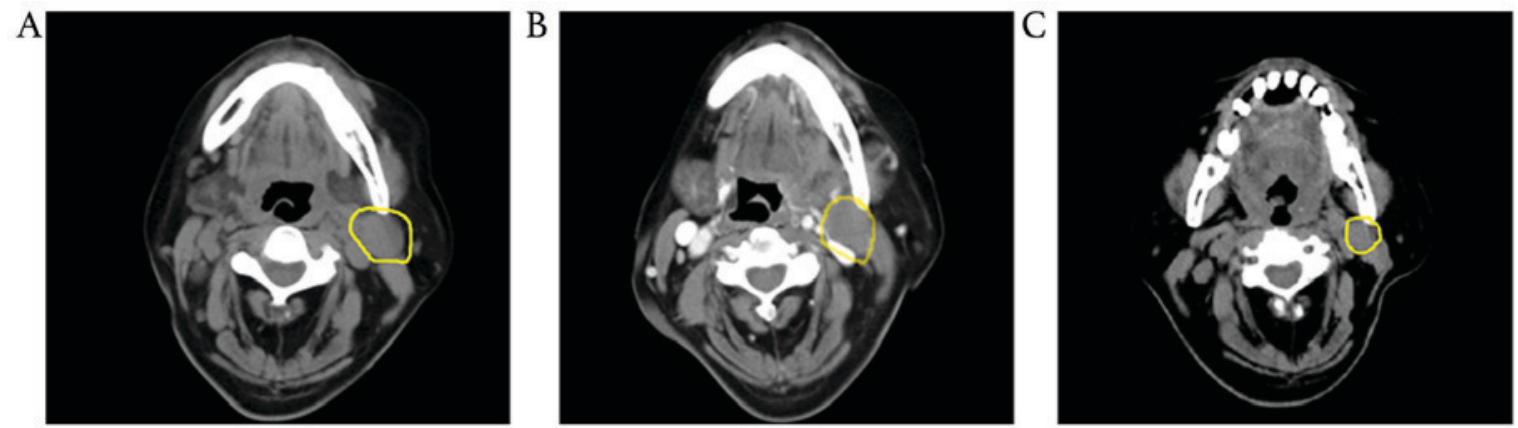

Figure 3. Contrast-enhanced computed tomography (CECT) scans of the enlarged cervical lymph node obtained in (A) June 2014, (B) August 2014, and (C) April 2015. The largest diameter of the cervical enlarged lymph node did not decrease.

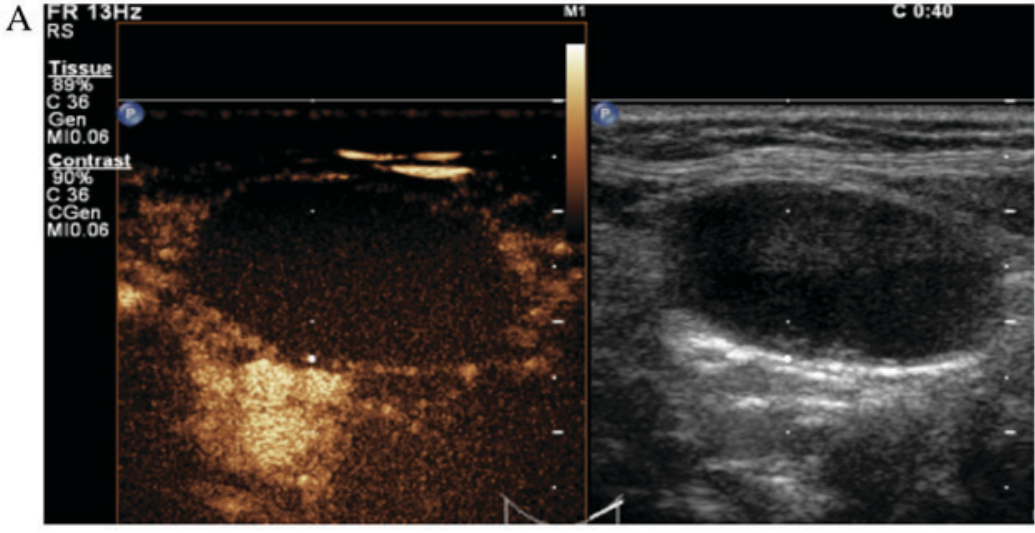

$\mathrm{B}$

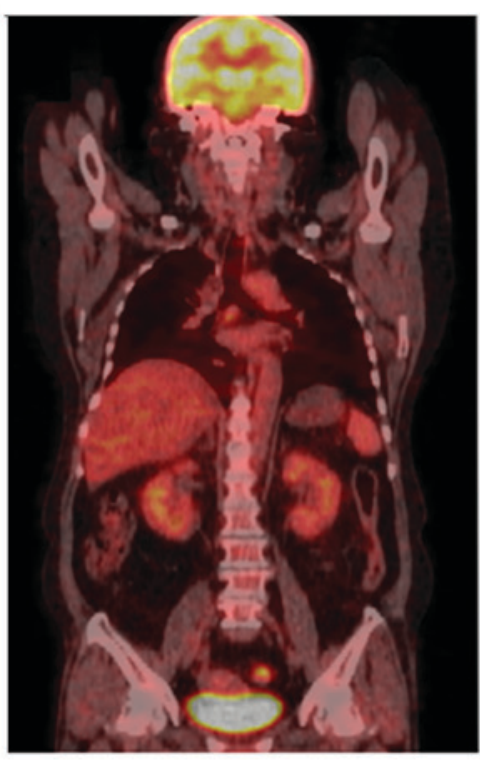

$\mathrm{D}$

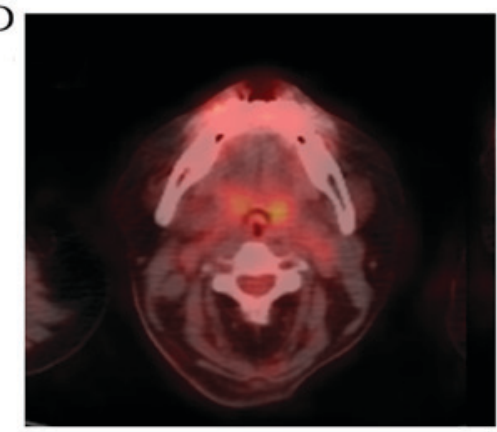

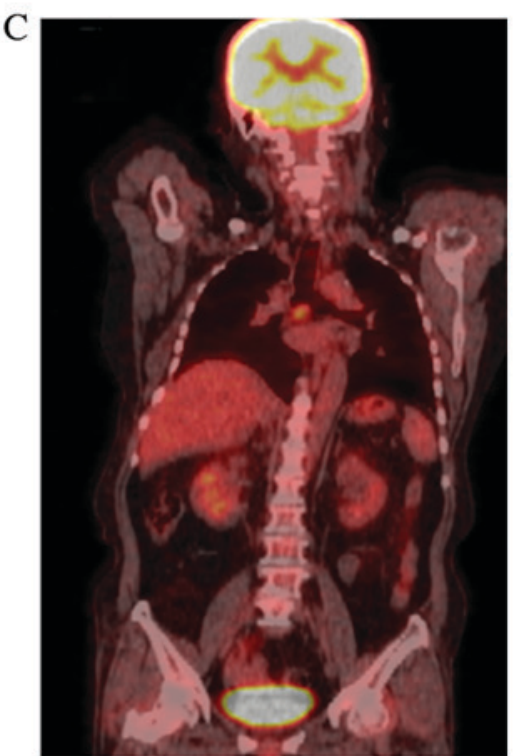

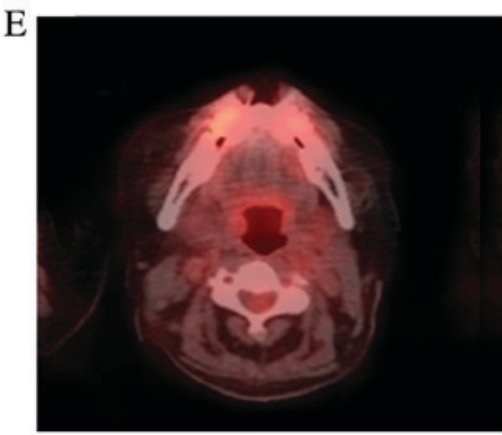

Figure 4. (A) Contrast-enhanced ultrasound (CEUS) showed no enhancement in the cervical lesion. It suggested no blood perfusion in the enlarged cervical lymph node. (B-E) Coronal and transaxial PET/CT images did not show increased ${ }^{18} \mathrm{~F}$-FDG uptake at previously tumorous lesions in September (B and C) and December (D and E), respectively. 
at baseline and after two and four cycles results in tremendous economic stress to patients, especially to those without medical insurance. Because most PET/CT devices are installed at metropolitan tertiary care centers, patients in countrywide hospitals have limited access to it. Thus, in countrywide hospitals and some metropolitan tertiary care centers, CECT is commonly used in clinical practice to assess treatment response. Without metabolic information from tumor lesions, CECT can only provide morphological information, whereas when a patient responds well to chemotherapy, there are changes in the tumor metabolism that start before a visible decrease in size can be detected, which leads to a false positive result in the treatment response assessment with CECT imaging. This inaccuracy will underestimate the effectiveness of current chemotherapy; in some inappropriate situations, a more intense chemotherapy regimen will be adopted. In addition to the disadvantages described earlier, a more intense chemotherapy regimen brings patients more psychological stress and discomfort.

CEUS is minimally invasive, easy to use, inexpensive, and allows for reproducible measurements without adverse effects. It can be performed at the bedside through the injection of highly echogenic microbubbles able to enter the microcirculation, which allows for real-time imaging of organ and lesion blood perfusion. It is frequently employed in clinical practice, including early posttransplant evaluation of graft perfusion and detection of imcomplete liver percutaneous radiofrequency ablation (19-24). In recent studies, CEUS has been utilized in the differential diagnosis of liver lymphoma, cervical lymphadenopathy and superficial lymph nodes $(12,25-27)$. CEUS could exhibit comparable results to CECT in response assessment. In the literature, there is no evidence related to the use of CEUS for detecting blood perfusion, tumor growth, and treatment response. Prospective studies are required to assess whether the disappearance of blood flow can be correlated to lymphoma growth status.

In conclusion, CEUS seems to be an efficient, feasible and minimally invasive, but not substitutive imaging option that provides complementary results to CECT in the therapeutic assessment of DLBCL.

\section{Acknowledgements}

The present study was funded by the National Natural Science Foundation of China (81501488) and the Chengdu Science and Technology Agency (2014-HM01-00021-SF).

\section{References}

1. Armitage JO and Weisenburger DD: New approach to classifying non-Hodgkin's lymphomas: Clinical features of the major histologic subtypes. Non-Hodgkin's Lymphoma classification project. J Clin Oncol 16: 2780-2795, 1998.

2. Feugier P, Van Hoof A, Sebban C, Solal-Celigny P, Bouabdallah R, Fermé C, Christian B, Lepage E, Tilly H, Morschhauser F, et al: Long-term results of the R-CHOP study in the treatment of elderly patients with diffuse large B-cell lymphoma: A study by the Groupe d'Etude des Lymphomes de l'Adulte. J Clin Oncol 23: 4117-4126, 2005.

3. Fuertes S, Setoain X, Lopez-Guillermo A, Carrasco JL, Rodríguez S, Rovira J and Pons F: Interim FDG PET/CT as a prognostic factor in diffuse large B-cell lymphoma. Eur J Nucl Med Mol Imaging 40: 496-504, 2013.

4. Meignan M, Itti E, Gallamini A and Haioun C: Interim 18F-fluorodeoxyglucose positron emission tomography in diffuse large B-cell lymphoma: Qualitative or quantitative interpretation-where do we stand? Leuk Lymphoma 50: 1753-1756, 2009.
5. Haioun C, Itti E, Rahmouni A, Brice P, Rain JD, Belhadj K, Gaulard P, Garderet L, Lepage E, Reyes F and Meignan M: [18F]fluoro-2-deoxy-D-glucose positron emission tomography (FDG-PET) in aggressive lymphoma: An early prognostic tool for predicting patient outcome. Blood 106: 1376-1381, 2005.

6. Mikhaeel NG, Hutchings M, Fields PA, O'Doherty MJ and Timothy AR: FDG-PET after two to three cycles of chemotherapy predicts progression-free and overall survival in high-grade non-Hodgkin lymphoma. Ann Oncol 16: 1514-1523, 2005.

7. Hutchings M, Mikhaeel NG, Fields PA, Nunan $T$ and Timothy AR: Prognostic value of interim FDG-PET after two or three cycles of chemotherapy in Hodgkin lymphoma. Ann Oncol 16: 1160-1168, 2005.

8. Cashen AF, Dehdashti F, Luo J, Homb A, Siegel BA and Bartlett NL: 18F-FDG PET/CT for early response assessment in diffuse large B-cell lymphoma: Poor predictive value of international harmonization project interpretation. J Nucl Med 52: 386-392, 2011.

9. Allard CB, Coret A, Dason S, Tajzler C, Shayegan B, Matsumoto ED and Kapoor A: Contrast-enhanced ultrasonography for surveillance of radiofrequency-ablated renal tumors: A prospective, radiologist-blinded pilot study. Urology 86: 1174-1178, 2015.

10. Li CX, Lu Q, Huang BJ, Xue LY, Yan LX, Wen JX and Wang WP: The value of contrast-enhanced ultrasound (CEUS) in detecting minute renal cell carcinoma. Discov Med 18: 179-188, 2014.

11. Xue LY, Lu Q, Huang BJ, Li Z, Li CX, Wen JX and Wang WP: Papillary renal cell carcinoma and clear cell renal cell carcinoma: Differentiation of distincthistological types with contrast-enhanced ultrasonography. Eur J Radiol 84: 1849-1856, 2015.

12. Trenker C, Kunsch S, Michl P, Wissniowski TT, Goerg K and Goerg C: Contrast-enhanced ultrasound (CEUS) in hepatic lymphoma: Retrospective evaluation in 38 cases. Ultraschall Med 35: 142-148, 2014.

13. Li W, Liu G, Wang W, Wang Z, Huang Y, Xu Z, Xie X and Lu M: Real-time contrast enhanced ultrasound imaging of focal splenic lesions. Eur J Radiol 83: 646-653, 2014.

14. Yang DH, Ahn JS, Byun BH, Min JJ, Kweon SS, Chae YS, Sohn SK, Lee SW, Kim HW, Jung SH, et al: Interim PET/CT-based prognostic model for the treatment of diffuse large B cell lymphoma in the post-rituximab era. Ann Hematol 92: 471-479, 2013.

15. Itti E, Meignan M, Berriolo-Riedinger A, Biggi A, Cashen AF Véra P, Tilly H, Siegel BA, Gallamini A, Casasnovas RO and Haioun C: An international confirmatory study of the prognostic value of early PET/CT in diffuse large B-cell lymphoma: Comparison between Deauville criteria and DeltaSUVmax. Eur J Nucl Med Mol Imaging 40: 1312-1320, 2013.

16. Gonzalez-Barca E, Canales M, Cortés M, Vidal MJ, Salar A, Oriol A, Bargay J, Bello JL, Sánchez JJ, Tomás JF, et al: Predictive value of interim ${ }^{18} \mathrm{~F}$-FDG-PET/CT for event-free survival in patients with diffuse large B-cell lymphoma homogenously treated in a phase II trial with six cycles of R-CHOP-14 plus pegfilgrastim as first-line treatment. Nucl Med Commun 34: 946-952, 2013.

17. Iams W and Reddy NM: Consolidative autologous hematopoietic stem-cell transplantation in first remission for non-Hodgkin lymphoma: Current indications and future perspective. Ther Adv Hematol 5: 153-167, 2014

18. Dahi PB, Tamari R, Devlin SM, Maloy M, Bhatt V, Scordo M, Goldberg J, Zelenetz AD, Hamlin PA, Matasar MJ, et al: Favorable outcomes in elderly patients undergoing high-dose therapy and autologous stem cell transplantation for non-Hodgkin lymphoma. Biol Blood Marrow Transplant 20: 2004-2009, 2014.

19. Mauri G, Porazzi E, Cova L, Restelli U, Tondolo T, Bonfanti M, Cerri A, Ierace T, Croce D and Solbiati L: Intraprocedural contrast-enhanced ultrasound (CEUS) in liver percutaneous radiofrequency ablation: Clinical impact and health technology assessment. Insights Imaging 5: 209-216, 2014.

20. Pichler R, Heidegger I, Aigner F, Bösmüller C, Schneeberger S, Maglione M, Schäfer G, Steiner $\mathrm{H}$ and Horninger W: De novo renal cell carcinoma in a kidney allograft with focus on contrast-enhanced ultrasound. Urol Int 93: 364-367, 2014.

21. McKay H, Ducharlet K, Temple F and Sutherland T: Contrast enhanced ultrasound (CEUS) in the diagnosis of post-partum bilateral renal cortical necrosis: A case report and review of the literature. Abdom Imaging 39: 550-553, 2014.

22. Sanchez K and Barr RG: Contrast-enhanced ultrasound detection and treatment guidance in a renal transplant patient with renal cell carcinoma. Ultrasound Q 25: 171-173, 2009.

23. Badea R, Chiorean L, Mitre C, Botar-Jid C and Caraiani C: Spontaneous retroperitoneal and subcapsular liver hematoma. The diagnostic contribution of CT, US and CEUS. Case report. Med Ultrason 15: 157-160, 2013. 
24. Cao BS, Li L, Li YX and Liang YM: Assessment of abnormal bowel perfusion using contrast-enhanced ultrasonography after small bowel transplantation: A case report. J Clin Ultrasound 41: 370-372, 2013

25. Abe H, Kamimura K, Mamizu M, Shibazaki Y, Ishiguro T, Katada S, Nishiyama YK, Takahashi Y, Hatano YY, Mizuno K, et al: Early diagnosis of hepatic intravascular lymphoma: A case report and literature review. Intern Med 53: $587-593,2014$
26. Fodor D, Pascu I, Pop S and Poanta L: The utility of elastography and CEUS for the differentiation between benign and malignant cervical lymphadenopathy. Three cases report. Med Ultrason 15: 63-66, 2013.

27. de Stefano G, Scognamiglio U, Di Martino F, Parrella R, Scarano F, Signoriello G and Farella N: The role of CEUS in characterization of superficial lymph nodes: A single center prospective study. Oncotarget 7: 52416-52422, 2016. 